were not classified by currently accepted criteria, ${ }^{3}$ but $C$ fetus has probably been responsible for 10 previously reported human abortions. ${ }^{4}$ Thermophilic campylobacters, including $C$ jejuni and $C$ coli, have been identified in 11 previously reported cases, ${ }^{45}$ but many of these were not investigated with the hippurate hydrolysis test that is required for further identification.

Clinical features of this case are similar to those previously reported, with abortion occurring in the second trimester, and associated with a mild maternal illness consisting of diarrhoea and fever.

Histological examination of the placenta was not included for many of the previously reported cases, but the existing descriptions are all similar, with perivillous fibrin deposition, an inflammatory infiltrate of neutrophil polymorphonuclear leucocytes, and microabscess formation. In sharp contrast to the bovine cases, there has been no evidence of chorioamnionitis in all the cases reported to date. The presence of this feature in this case raises the possibility of ascending infection rather than septicaemic spread, and this may therefore be an alternative route of infection in humans.
The recognition of Campylobacter species as a cause of diarrhoea was delayed because they have fairly exacting cultural requirements and will not grow with the incubation and media regimens that are commonly used. They can, however, be reliably cultured and identified under suitable conditions. This realisation has led to their frequent detection in faecal specimens.

The finding of unexplained villitis with negative bacteriological investigations in spontaneous abortion is not uncommon, and it is possible that some of these cases are in fact due to Campylobacter species.

1 Skirrow MB. Campylobacter enteritis: a new disease. Br Med J 1977;ii:9-11.

2 Coid CR, Fox H. Campylobacter as placental pathogens. Placenta 1983;4:295-305.

3 Karmali MA, Skirrow MB. Taxonomy of the genus Campylobacter. In: Butzler JP, ed. Campylobacter infection in man and animals. Boca Raton, Florida: CRC press,

4 Simor AE, Karmali MA, Jadaviji T, Roscoe M. Abortion and perinatal sepsis associated with Campylobacter infection. Rev Infect Dis 1986;8:397-402.

5 Moscuna M, Gross Z, Korenblum R, et al. Septic abortion due to campylobacter jejuni. Eur J Clin Microbiol Infect Dis 1989;8:800-1.

\title{
AgNOR counts and determination of malignancy in stromal tumours of the stomach and small intestine
}

\author{
T W Beer, D C Rowlands, J Crocker
}

Department of Histopathology, East Birmingham Hospital, Bordesley Green East, Birmingham, B9 5ST $T$ W Beer D C Rowlands J Crocker

Correspondence to: Dr T W Beer, Registrar in Histopathology, Department of Histopathology, Birmingam Genera Hospital, Steelhouse Lane, Birmingam, $\mathrm{B} 4 \mathrm{NH}$.

Accepted for publication 4 July 1991

\begin{abstract}
Twenty four primary stromal tumours of the stomach and small intestine were investigated by silver staining of interphase nucleolar organiser regions (AgNORs) in an attempt to obtain an objective criterion for prediction of malignant tumour behaviour. Malignant tumours tended to have higher AgNOR counts than their benign counterparts, but this increase was small and there was some overlap between the two groups. There was a correlation between the mean AgNOR count and the mitotic count. There was no correlation between tumour size and these two measurements. This study suggests that in these stromal tumours the AgNOR count is not a useful independent predictor of malignant behaviour.
\end{abstract}

Definitive histopathological distinction between benign and malignant stromal tumours of the gut is often difficult. It is widely accepted that the most discriminative criterion in distinguishing these groups is the mitotic count. ${ }^{12}$ The ability to predict an individual tumour's behaviour is of great importance in planning the treatment and continuing care of the patient.

It has been proposed that an increased number of actively transcribing nucleolar organiser regions is a marker of malignancy ${ }^{3}$ and that staining these structures by the AgNOR technique may be useful in the diagnois and assessment of prognosis in some tumours. ${ }^{4}$ This study investigated whether the enumeration of AgNORs in stromal tumours of the stomach and small intestine is useful as a diagnostic method to distinguish benign from malignant 
tumours or if it is an independent predictor of prognosis.

\section{Methods}

All 29 surgical resection specimens of stromal tumours of the stomach and small intestine performed at East Birmingham Hospital over a 15 year period were reviewed. Four of these tumours were small and were incidental findings in specimens resected for other pathology. There was insufficient follow up on a fifth tumour which was also less than $1 \mathrm{~cm}$ in diameter. The 24 cases with adequate clinical and follow up data were studied further. Seventeen of these tumours were from the stomach and the remaining seven from the small bowel.

The patients' ages varied from 16 to 85 years (mean 62 years). There were 11 men and 13 women. Five of the gastric tumours and two of the small bowel tumours had been originally called malignant on the basis of their histological and clinical features. All of the original sections from these cases were reviewed.

Serial $3 \mu \mathrm{m}$ sections were cut from representative blocks of each tumour, and one section was stained with haematoxylin and eosin for assessment of the mitotic count. The number of mitoses in $\mathbf{4 0}$ high power fields was counted from the most cellular areas of each tumour using a Leitz Dialux 20EB microscope at a magnification of $\times 400$ ( 1 high power field area $=0.159 \mathrm{~mm}^{2}$ ). Sections of the tumours were stained with the standard AgNOR technique for nucleolar organiser regions ${ }^{3}$ and a modified AgNOR method that used polyethylene glycol 20000 as the colloidal developer. ${ }^{5}$ The number of AgNORs in 200 tumour cell nuclei from the most cellular areas of each tumour were counted at a magnification of $\times 1000 .{ }^{6}$ The mean number of AgNORs per nucleus was calculated for each specimen.

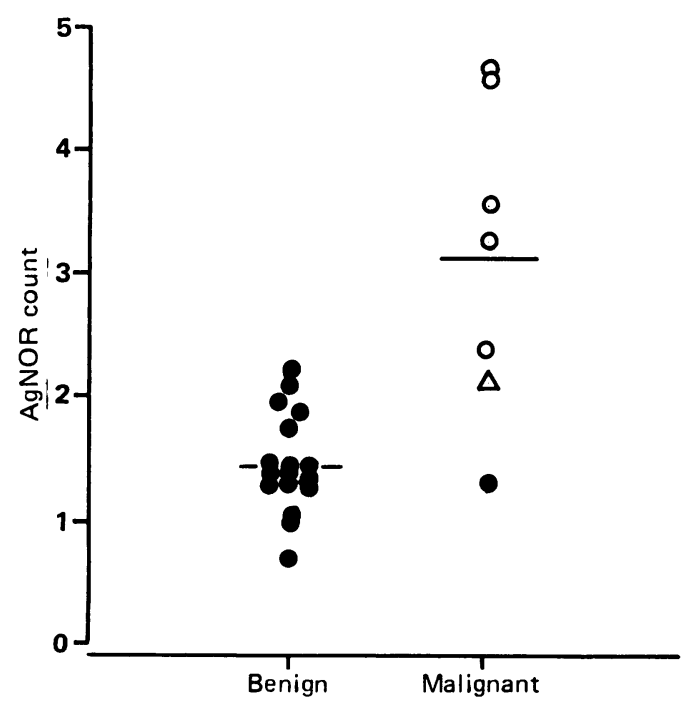

Scatter diagram to show mean AgNOR count per nucleus for the benign and malignant smooth muscle tumours. The bars indicate the mean value for each group. Open circles indicate the patients who died of their tumour. The open triangle indicates the patient with recurrent disease after six years of follow up.

\section{Results}

Of the seven patients with tumours that had originally been labelled as malignant, four had died of their tumour and another had had a local recurrence with hepatic metastases six years after the primary resection. The two other patients were disease free after at least a four year follow up, but review of their tumours showed clear evidence of malignancy with a raised mitotic rate, large tumour size, or local invasion. None of the 17 patients whose tumours had been called benign showed any evidence of recurrence after at least a four year follow up.

The mean number of mitoses per 10 high power fields in the benign tumours was 0.71 , and in the malignant group, $5 \cdot 0$. The difference in the medians between the two groups was 5 (95\% CI 1 to 6). There was overlap between the groups: two of the malignant tumours had mitotic counts in the range that included all of the benign tumours (0 to 2$)$.

AgNOR staining of the tumours with the standard AgNOR technique ${ }^{3}$ resulted in unacceptable non-specific precipitate on the dense stroma often found in these tumours, and this made accurate AgNOR counting impossible in many cases. In contrast, with the modified AgNOR staining method, all tumours gave well defined AgNOR staining with minimal background staining and precipitate. The mean number of AgNORs per nucleus for the benign tumours was 1.48 and for the malignant tumours $3 \cdot 16$ (figure). The difference between the medians of the two groups was 1.69 (95\% CI 0.71 to 2.77 ). There was, however, overlap between the two groups: three of the seven malignant tumours had AgNOR counts similar to the range seen in the benign group. When the maximum number of AgNORs in each tumour was considered there was a similar degree of overlap between benign and malignant groups. There was a correlation between the AgNOR count and the mitotic count $(\mathrm{r}=0 \cdot 74,95 \% \mathrm{CI}$ 0.48 to 0.88 ).

Of the four patients who died of malignant disease, all had mitotic counts for 5 to 10 per high power fields. One of these tumours had an AgNOR count within the range shown by the benign tumours. There was no difference in the range of AgNOR counts between gastric and small bowel tumours. No correlation was found between tumour size and the AgNOR or mitotic counts.

\section{Discussion}

The malignant tumours tended to have higher AgNOR counts than their benign counterparts, but the difference between these values in the two groups was small and there was a degree of overlap between them. These results are similar to those of Sinn $\mathrm{et} \mathrm{al}^{7}$. In contrast to their findings, however, we found that the interphase AgNOR count seemed to correlate with the mitotic count.

In this study the AgNOR count was no more discriminating than the mitotic count in distinguishing benign from malignant stromal tumours. The two parameters are probably related, in keeping with the opinion that the 
AgNOR counts represent a marker of proliferative activity. ${ }^{4}$ Although there are problems and limitations to mitotic counting, ${ }^{8}$ this method has become widely accepted in the routine assessment of stromal tumours of the gut. $^{12}$ The mitotic count can be performed more quickly and easily than AgNOR staining and uses routinely available haematoxylin and eosin stained sections. There is also a wider variation in the mitotic count than there is for the AgNOR count. Because there is only a small difference in AgNOR count between benign and malignant tumours, it is unlikely that the technique has any practical use for the diagnosis or prognostication in these neoplasms.

We thank Mr B Simms for photographic assistance and Dr EJ Lazda for help with graphics.
1 Evans HL. Smooth muscle tumours of the gastrointestinal tract. A study of 56 cases followed for a minimum of 10 years. Cancer 1985;56:2242-50.

2 Ranchod M, Kempson RL. Smooth muscle tumours of the gastrointestinal tract and retroperitoneum. A pathologic gastrointestinal tract and retroperitoneum. A pathologic
analysis of 100 cases. Cancer 1977;39:255-62.

3 analysis of 100 cases. Cancer 1977;39:255-62.

, Menager $M$, Jeannesson $P$, Himber G, Pigeon $F$ Adnett JJ. Improvement in the staining and in the visualization of the argyrophilic proteins of the nucleolar organizer region at the optical level. Histochem $J$ 1986;18:5-14.

4 Crocker J. Nucleolar organizer regions. In: Underwood JCE, ed. Current topics in pathology. Vol 82: Pathology of the nucleus. Berlin: Springer-Verlag, 1990:91-149.

5 Rowlands DC, Crocker J, Ayres JG. Silver staining of nucleolar organizer region associated proteins using polyethylene glycol as the protective colloidal developer. Histochem J 1990;22:555-9.

6 Crocker J, Boldy DAR, Egan MJ. How should we count AgNORs? Proposals for a standardized approach. J Pathol 1990;158:185-88.

7 Sinn HP, Lehnert T, Kandetzki C, Waldherr R. Nucleolar organizer regions in myogenic stromal tumours of the stomach. Virchows Arch (Pathol Anat) 1989;415:317-21.

8 Quinn CM, Wright NA. The clinical assessment of proliferation and growth in human tumours: evaluation of methods and applications as prognostic variables. J Pathol 1990;160:93-102.

\title{
Salmonella osteomyelitis in aplastic anaemia after antilymphocytic globulin and steroid treatment
}

\author{
S Allard, J O’Driscoll, A Laurie
}

Department of Haematology, St George's Hospital. Blackshaw Road, London SW17 0QT S Allard

A Laurie

Department of Microbiology

J O'Driscoll

Correspondence to: Dr S Allard

Accepted for publication 4 July 1991

\begin{abstract}
A 22 year old patient with severe aplastic anaemia responded to antilymphocytic globulin but developed recurrent fever despite treatment with steroids and then antibacterial, antifungal, and antituberculous drugs. There was progression, with severe joint pains and immobility associated with radiological evidence of a symmetrical destructive process. A bone marrow specimen showed no evidence of malignancy, and cultures of blood, urine, and stool were negative but enriched broth cultures of an open biopsy specimen of the humeral head grew Salmonella enteriditis phage type 4. Treatment with ciprofloxacin resulted in considerable symptomatic improvement: a total of 12 months of treatment is planned. Salmonella osteomyelitis, particularly with this unusual pattern of disease, has not previously been described in aplastic anaemia.
\end{abstract}

\section{Case report}

Bone and joint infections due to Salmonella are uncommon, although various predisposing conditions are recognised, particularly sickle cell anaemia. ${ }^{1-3}$ We describe a patient with aplastic anaemia who responded well to treatment with antilymphocytic globulin (ALG) but who developed severe Salmonella enteriditis (type 4) osteomyelitis.

A 22 year old Greek woman presented with anaemia and thrombocytopenia (haemoglobin $5 \mathrm{~g} / \mathrm{dl}$; platelets $42 \times 10^{9} / 1$; white cell count $3.3 \times 10^{9} / 1$, with $46 \%$ neutrophils, $48 \%$ lymphocytes, and $6 \%$ monocytes), and investigations confirmed idiopathic aplastic anaemia. She received $15 \mathrm{mg} / \mathrm{kg}$ equine ALG (Merrieux) daily for five days infused into the subclavian vein via a line inserted into the antecubital fossa. Five days later a typical syndrome of serum sickness developed with fever, rash, and arthralgia and this responded promptly to hydrocortisone.

High fever with negative blood cultures 